This is the post peer-review accepted manuscript of:

D. Dardari and F. Guidi, "Direct position estimation from wavefront curvature with single antenna array," 2018 8th International Conference on Localization and GNSS (ICL-GNSS), Guimaraes, 2018, pp. 1-5.

doi:

https://doi.org/10.1109/ICL-GNSS.2018.8543121

The published version is available online at:

http://ieeexplore.ieee.org/stamp/stamp.jsp?tp=\&arnumber $=8543121$ \&isnumber $=8440892$

C 2018 IEEE. Personal use of this material is permitted. Permission from IEEE must be obtained for all other uses, in any current or future media, including reprinting/republishing this material for advertising or promotional purposes, creating new collective works, for resale or redistribution to servers or lists, or reuse of any copyrighted component of this work in other works 


\title{
Direct Position Estimation from Wavefront Curvature with Single Antenna Array
}

\author{
Davide Dardari, Francesco Guidi \\ DEI, University of Bologna, via Venezia 52, I-47521 Cesena (FC), Italy. \\ e-mail: \{davide.dardari,f.guidi\}@unibo.it
}

\begin{abstract}
In this paper we investigate the possibility to perform direct positioning by retrieving information from the wavefront curvature. Despite such an approach has been considered in the past at microwave and acoustic frequencies using extremely large antennas, it is of interest to investigate its potential exploitation at $\mathbf{m m}$-wave with practical size antennas in the context of next $5 \mathrm{G}$ systems. Thus, here we first consider a dedicated model to gather the source position information from the wavefront curvature for different array architectures, i.e., traditional and lens-based arrays, and successively we derive the maximum likelihood estimator to investigate the attainable performance. Results, obtained for different number of antennas, i.e., for different array apertures, confirm the possibility to achieve interesting positioning performance using a single antenna array with limited dimensions.
\end{abstract}

Index Terms - Wavefront curvature, direct positioning, massive array, lens array, mm-wave

\section{INTRODUCTION}

Next fifth generation (5G) of mobile wireless communications foresees, among all the technologies, the joint use of millimeter-waves (mm-wave) and massive arrays to enable the integration of arrays with a large number of antennas into small areas. By enabling such an architecture capable to realize near-pencil beam antennas, it becomes feasible to boost communication and localization capabilities at an unprecedented scale [1]-[3]. For example, in [4] a joint energy-detection and massive array design has been conceived to perform target localization and environment mapping. To further reduce the complexity, analog and hybrid analog-digital schemes have been investigated [5], and the attainable fundamental localization limits using different array configurations have been investigated [6].

Recently, it has also been studied the possibility to exploit electromagnetic (EM) lens-based massive arrays operating at $\mathrm{mm}$-wave as a promising solution for drastically reducing the overall system complexity [7]. In fact, by adopting a lens to collimate the beams in precise directions, it is possible to spatially discriminate signals in the analog domain [7]-[9]. Consequently, thanks to the lens, there is a unique relation between the incident and the output angles of the impinging and refracted waves, respectively. This operation allows the reduction of the number of antennas with respect to traditional massive arrays, and to move from discrete beamforming architectures towards continuous-aperture-phased arrays.

Positioning approaches based on antenna arrays usually rely on a joint angle-of-arrival (AOA) and time-of-arrival (TOA) estimation process, the latter requiring multiple interactions between transmitter and receiver as well as an extremely precise system synchronization.

To overcome such a two-step based approach, a possible direct solution is to gather the transmitter position information from the wavefront curvature. In fact, whenever the planar approximation becomes not valid, the position information can be inferred from the waveform curvature without the need of Tx-Rx synchronization. This concept is not new: in [10], the curvature information has been exploited, with a moving source approaching to the receiver so that, entering in the Fresnel region, the incoming wave cannot be regarded as plane anymore. In [11], an approach for direct wireless positioning with narrowband signals with multi-tone signalling and multi-arrays is described. In [12], a MUSIC-based method is proposed to retrieve information from the wavefront curvature, and an extensive analysis on the attainable fundamental localization limits has been derived in near-field propagation conditions [13]. A deep investigation using acoustic waveforms has been carried out in [14], [15].

All previous studies can be applicable to those situations where the waveform curvature is significant with respect to the antenna aperture in relation to the wavelength. This is true, for example, exploiting acoustic waves or at microwave only considering very short distances or using very large (often not practical) antennas. With the introduction of mm-wave technology, the possibility to perform one-shot direct positioning by exploiting the waveform curvature becomes of large interest. In particular, thanks to the extremely small wavelength, direct positioning is in principle possibile even with antenna arrays with limited aperture .

In this paper we investigate the localization capability of a $\mathrm{mm}$-wave source using a single antenna array with the purpose to understand the potentialities of the wavefront curvature approach in next generation of mw-wave positioning systems. A comparison accounting for a different number of antennas in the single arrays, as well as for implementations using or not an EM lens, is also carried out by means of a maximum likelihood (ML) estimator.

The rest of the paper is organized as follows. Sec. II contains insights on how to gather position information from the signal wavefront when classical arrays are used, whereas Sec. III reports considerations when lens arrays are exploited. Sec. IV reports the ML estimator derivation, while in Sec. V results are reported. Finally, conclusions are drawn in Sec. VI. 


\section{POSITION INFORMATION IN THE WAVEFRONT CURVATURE}

Consider a transmitting source located at position $\mathbf{p}$, which is at distance $d$ from the reference point of the RX, indicated with 0 , and denote with $\theta$ the incident angle, formed with 0 , as indicated in Fig. 1. The extra distance traveled by the EM wave to reach the generic coordinate $z$ of the RX antenna aperture (please refer to Fig. 1) is given by

$$
a(z, \mathbf{p})=-d+d \sqrt{1+\frac{z^{2}}{d^{2}}+2 \cdot \frac{z}{d} \sin \theta}
$$

that, differently from classical antenna arrays with planar wavefront, does not depend only on the AOA $\theta$ but also on the distance $d$, i.e., on the position p. Consequently, the equivalent complex baseband signal received in the $z$ th position of the antenna aperture is

$$
s(z, \mathbf{p})=h(z, \mathbf{p}) x_{0}
$$

where $h(z, \mathbf{p})$ is given by

$$
h(z, \mathbf{p})=e^{-j 2 \pi f_{0} \tau(z, \mathbf{p})}
$$

with $f_{0}$ being the central frequency and

$$
\tau(z, \mathbf{p})=\frac{a(z, \mathbf{p})}{c}
$$

with $c$ being the speed of light, whereas the signal $x_{0}$ takes the form

$$
x_{0}=A_{\mathrm{pl}} e^{-j \chi}
$$

where $A_{\mathrm{pl}}$ denotes the received signal amplitude, and $\chi \sim$ $\mathcal{U}[0,2 \pi)$ is uniformly distributed between 0 and $2 \pi$. Thus, $\chi$ includes the complete uncertainty on the received signal phase, since the transmitter and receiver are supposed to be not not synchronized and no information can be retrieved from the TOA of the received signal. In addition, note that, if $z \ll d$, then $a(z, \mathbf{p}) \approx \frac{z^{2}}{2 d}+z \sin \theta$, where the second term refers to the traditional array phase term containing AOA information, whereas the first term includes information on the source distance.

\section{A. Antenna Array Model}

Now we account for an array with $N_{\mathrm{A}}$ antennas placed at an inter-distance of $\lambda / 2$, with $\lambda$ indicating the wavelength, so that signals are spatially sampled at Nyquist (considerations according to the spatial position of the antennas are reported in the following), i.e, $r_{n}=r(n \lambda / 2)$. In additive white Gaussian noise (AWGN) scenario, the RX signal at the $n$th antenna element is

$$
r_{n}=s_{n}(\mathbf{p})+w_{n}=h_{n}(\mathbf{p}) \cdot x_{0}+w_{n}
$$

where $w_{n}$ is the receiver noise at the $n$th antenna, $h_{n}(\mathbf{p})$ is the coefficient relative to the $n$th antenna given by

$$
h_{n}(\mathbf{p})=h(n \lambda / 2, \mathbf{p})=e^{-j 2 \pi f_{0} \tau\left(n \frac{\lambda}{2}, \mathbf{p}\right)}
$$

and $s_{n}(\mathbf{p})=s(n \lambda / 2, \mathbf{p})$. Thus, differently from classical arrays where the phase contains information only on the AOA,

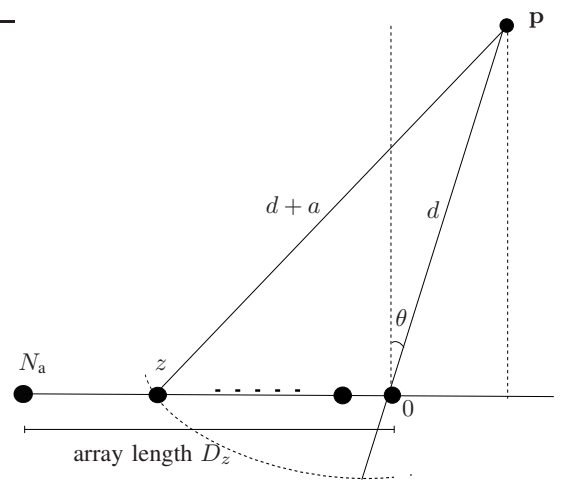

Fig. 1. Considered scenario, where the information on the position $\mathbf{p}$ is retrieved from the curvature wavefront.

here the information on $\mathbf{p}$ is embedded in the received signal. In this way, by properly designing the receiver, it is possible to directly infer the position of the transmitter avoiding a two-step approach based on the joint TOA-AOA measurement and on a prior synchronization phase to align the source and receiving array clocks.

1) Inter-Antenna Spacing with Planar Wavefront: In accordance with the state-of-the-art, in order to avoid ambiguities (i.e., aliasing), signals have to be spatially sampled by respecting the Shannon theorem in the spatial domain. To that purpose, assuming uniform spacing among antennas, we consider the term containing information on the AOA, namely $\Delta z_{\phi}=z_{n+1}-z_{n}$, with $n=0, \ldots, N_{\mathrm{A}}-1$, and $z_{n}$ being the distance of the $n$th antenna from the reference point 0 . According to [16], the maximum inter-element spacing is

$$
\Delta z_{\phi} \leq \frac{\lambda}{2}
$$

which is the classical constraint to be respected when placing antennas in arrays.

2) Inter-Antenna Spacing with Spherical Wavefront: Consider now the inter-antenna distance, namely $\Delta z_{\mathbf{p}}=z_{n+1}-$ $z_{n}$, with $n=0, \ldots, N_{\mathrm{A}}-1$, for which the same constraint related to the position has to be satisfied to avoid aliasing, i.e.,

$$
\frac{2 \pi}{\lambda}\left(\frac{1}{2 d} \cdot \Delta z_{\mathbf{p}}^{2}+\sin \theta \cdot \Delta z_{\mathbf{p}}\right) \leq \pi .
$$

In this case the constraint is expressed as a function of the distance from the source, and the solution of (9) is given by

$$
\Delta z_{\mathbf{p}} \leq d \sqrt{(\sin \theta)^{2}+\frac{\lambda}{d}}-d \sin \theta=\Delta z_{\mathbf{p}}^{\star} .
$$

From (10), given a constrain on the minimum distance of interest and the range of AOAs, one can determine the maximum inter-element distanze $\Delta z_{\mathbf{p}}$ to avoid aliasing in the space domain.

Note that this condition is close to the traditional one for $\Delta z_{\phi}$. In fact, assuming for example $\theta=\pi / 2$ and $\frac{\lambda}{d} \ll 1$, 


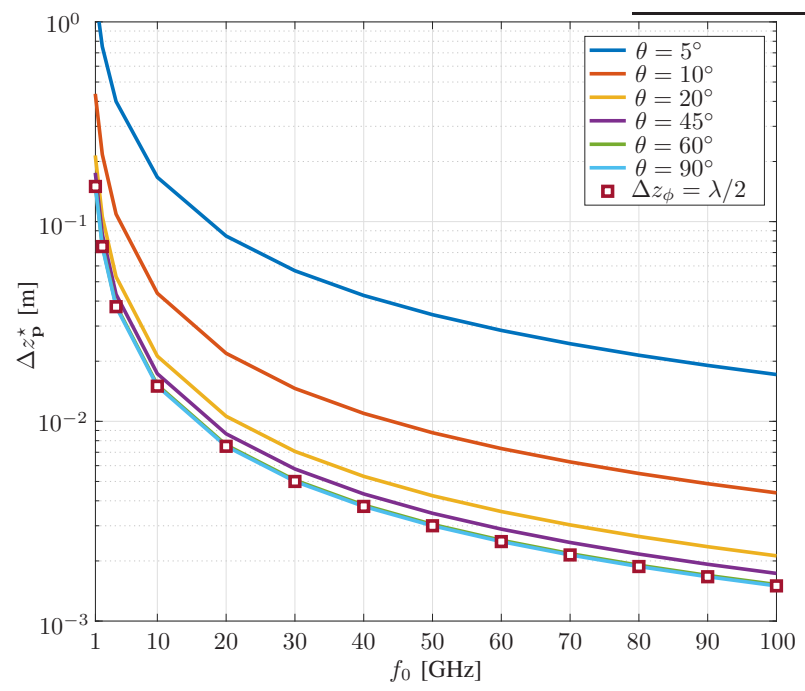

Fig. 2. Required inter-element distance $\Delta z_{\mathrm{p}}^{\star}$ when $d=30 \mathrm{~m}$ for different $\theta$, and comparison with the counterpart $\Delta z_{\phi}$.

through a Taylor series expansion, (10) can be approximated as $\sqrt{1+\frac{\lambda}{d}} \approx 1+\frac{\lambda}{2 d}$, that yields to

$$
\Delta z_{\mathbf{p}} \leq d\left(1+\frac{\lambda}{2 d}\right)-d=\frac{\lambda}{2} .
$$

Notably, (11) puts in evidence that the constraint on interelement spacing is dominated by the AOA term and not by the distance-dependent term.

In Fig. 2, we report the required inter-element distance when the distance $d$ is fixed, i.e., $d=30 \mathrm{~m}$, but different AOA are accounted for. In particular, it is evidenced that when considering the joint term $\Delta z_{\mathrm{p}}^{\star}$, the dominant contribution constraining the antenna spacing is still the one including the AOA information, as classically done in the array design for incident planar wavefront.

\section{LENS MODEL}

We now consider the generic scenario when a lens, as the one shown in Fig. 3, is introduced to collimate the impinging wave in specific directions. By following the guidelines given in [7], the array is equipped with $N_{\mathrm{A}}$ antennas located on the focal arc of the lens, lying on the $x z$-plane, with $\theta_{n} \in$ $[-\pi / 2, \pi / 2]$ representing the angle of the $n$th generic antenna element. Then, by defining $\tilde{\theta}_{n}=\sin \theta_{n}$, antenna elements are deployed so that $\tilde{\theta}_{n}$ results to be equally spaced in the interval $[-1,1]$ (critical sampling), i.e.,

$$
\tilde{\theta}_{n}=\frac{n \lambda}{D_{z}}=\frac{n}{\tilde{D}_{z}}
$$

where $\tilde{D}_{z}=D_{z} / \lambda$, with $D_{z}$ being the lens length along the $z$-axis. Notably, the analysis herein carried out is general and scales according to the operational frequency. Then, according to [7], the relation between $\tilde{D}_{z}$ and the required number of antennas $N_{\mathrm{A}}$ is

$$
N_{\mathrm{A}}=1+2\left\lfloor\tilde{D}_{z}\right\rfloor
$$

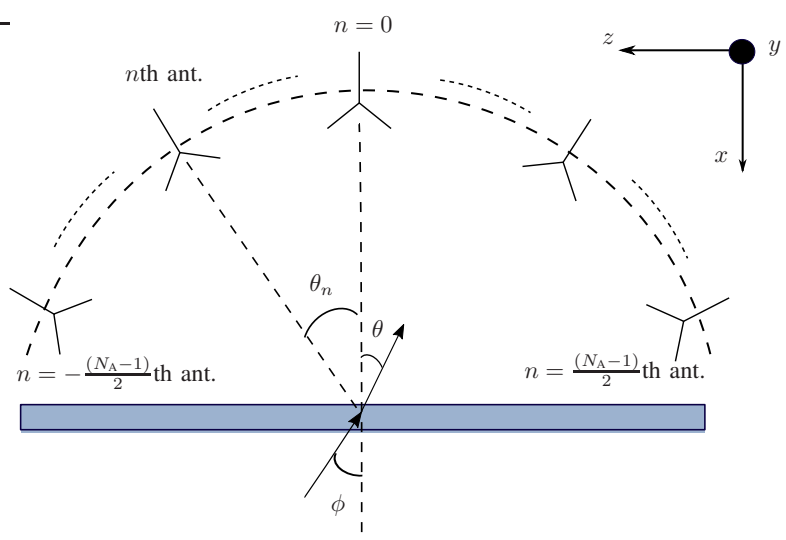

Fig. 3. Top-view of the EM lens massive array architecture [9].

with $\lfloor\cdot\rfloor$ denoting the largest integer no greater than its argument, so that (13) indicates the need of a higher number of antennas when the dimension $\tilde{D}_{z}$ of the antenna increases. Since it is $\theta_{n}=\arcsin \left(n / \tilde{D}_{z}\right)$, antenna elements are more densely located in the center of the system.

\section{A. Received Signal at Each Antenna}

In this section we derive the signal model accounting for the lens and the wavefront curvature. To this purpose, we extend the approach in [7], valid for plane waves, to our case. Thus, according to Fig. 3, we consider a 3D lens along the $y z$-plane. By accounting for a source located on the $x z$-plane, the signal received on the focal arc, at angle $\theta$, can be expressed as

$$
r(\theta)=\int_{-D_{y} / 2}^{D_{y} / 2} \int_{-D_{z} / 2}^{D_{z} / 2} s(y, z, \mathbf{p}) e^{-j \Psi} d z d y+w(\theta)
$$

where $\Psi$ is the dephasing term given by the lens, according to the analysis reported in [7] for an incident planar wavefront, and $s(y, z, \mathbf{p})$ is given by

$$
s(y, z, \mathbf{p})=\frac{x_{0}}{\lambda \sqrt{D_{y} D_{z}}} \cdot e^{j 2 \pi \frac{a(z, \mathbf{p})}{\lambda}} e^{j 2 \pi \frac{b(y, \mathbf{p})}{\lambda}}
$$

where in this case $x_{0}$ is the input signal arriving at the lens with $\mathrm{AOA} \phi$, and the normalization term $1 / \sqrt{D_{y} D_{z}}$ is chosen to guarantee that the overall power intercepted by the lens is proportional to its normalized aperture $\mathcal{A}=\left(D_{y} D_{z}\right) / \lambda^{2}$. In addition, note that $a(z, \mathbf{p})$ is given by $(1)$, whereas $b(y, \mathbf{p})$ is

$$
b(y, \mathbf{p})=-d+d \sqrt{1+\frac{y^{2}}{d^{2}}}
$$

due to the considered scenario, since the source lies on the $x z$-plane depicted in Fig. 3. Notably, in our scenario the term due to the curvature of the wavefront is now present and its distribution at the output of the lens can be used to retrieve the position information. Then, by indicating with $\tilde{z}=z / \lambda$ and $\tilde{\theta}=\sin \theta$, it is possible to write

$$
r(\tilde{\theta})=\int_{-\tilde{D}_{z} / 2}^{\tilde{D}_{z} / 2} \int_{-\tilde{D}_{y} / 2}^{\tilde{D}_{y} / 2} s(\tilde{y}, \tilde{z}, \mathbf{p}) e^{-j 2 \pi \tilde{z} \tilde{\theta}} d \tilde{y} d \tilde{z}+w
$$


with $w=w(\tilde{\theta})$, which gives

$$
\begin{aligned}
& r(\tilde{\theta})= \\
& \frac{x_{0}}{\sqrt{D_{y} D_{z}}} \int_{-\tilde{D}_{z} / 2}^{\tilde{D}_{z} / 2} \int_{-\tilde{D}_{y} / 2}^{\tilde{D}_{y} / 2} e^{j 2 \pi[\tilde{a}(\tilde{z}, \mathbf{p})+\tilde{b}(\tilde{y}, \mathbf{p})]} e^{-j 2 \pi \tilde{z} \tilde{\theta}} d \tilde{y} d \tilde{z}+w
\end{aligned}
$$

with

$$
\begin{aligned}
& \tilde{a}(\tilde{z}, \mathbf{p})=-\frac{d}{\lambda}+d \sqrt{\frac{1}{\lambda^{2}}+\frac{\tilde{z}^{2}}{d^{2}}+2 \frac{\tilde{z}}{\lambda d} \sin \theta} \\
& \tilde{b}(\tilde{y}, \mathbf{p})=-\frac{d}{\lambda}+d \sqrt{\frac{1}{\lambda^{2}}+\frac{\tilde{y}^{2}}{d^{2}}} .
\end{aligned}
$$

According to the antenna critical sampling previously described, the received signal can be written at each $n$th antenna placed in the focal arc as

$r_{n}=s_{n}(\mathbf{p})+w_{n}=$

$\frac{x_{0}}{\sqrt{D_{y} D_{z}}} \int_{-\tilde{D}_{z} / 2}^{\tilde{D}_{z} / 2} \int_{-\tilde{D}_{y} / 2}^{\tilde{D}_{y} / 2} e^{j 2 \pi[\tilde{a}(\tilde{z}, \mathbf{p})+\tilde{b}(\tilde{y}, \mathbf{p})]} e^{-j 2 \pi \tilde{z} \frac{n}{\tilde{D} z}} d \tilde{y} d \tilde{z}+w_{n}$.

Solving (20) allows to study the impact of wavefront curvature at the receiver antennas. Note that in case the wavefront is planar, the solution of (20) reduces the one given in [7].

In the following we derive the ML to retrieve the position information from the incoming signal wavefront, and successively we evaluate the attainable performance

\section{MAXimum LiKelihood}

The signals received by all the antennas are collected and post-processed together in order to estimate the distance of the source.

For the sake of simplicity, and in order to provide a solid benchmark for more practical estimators, we consider only the line-of-sight (LOS) component. Here we refer to a likelihood detector, where there is the maximization over the position $\mathbf{p}$ of the transmitter allowed by the curvature of the incoming wavefront and the unknown phase $\chi$. The likelihood function related to the position $\mathbf{p}$ and the unknown phase $\chi$ can be written as

$$
\Lambda(\mathbf{p}) \propto \prod_{n=1}^{N_{\mathrm{A}}} \exp \left\{-\frac{1}{2 \sigma^{2}}\left\|r_{n}-s_{n}(\mathbf{p})\right\|^{2}\right\}
$$

where $\sigma^{2}=N_{0} W$, with $N_{0}$ representing the noise power spectral density (PSD) at each antenna, and where we have made explicit the dependence of $s_{n}(\mathbf{p})$ on the position $\mathbf{p}$. Taking the logarithm and discarding all the terms that do not bring contribution for maximizing $\mathbf{p}$, the log-likelihood function reduces to

$$
l(\mathbf{p})=\sum_{n=1}^{N_{\mathrm{A}}} \Re\left\{r_{n} \cdot s_{n}^{*}(\mathbf{p})\right\} .
$$

Finally, the ML estimate of the distance can be expressed as

$$
\hat{\mathbf{p}}=\arg \max _{\mathbf{p}, \chi}[l(\mathbf{p})]
$$

that, in accordance with the previous derivation, yields to

$$
\hat{\mathbf{p}}=\arg \max _{\mathbf{p}, \chi}\left\{\sum_{n=1}^{N_{\mathrm{A}}} \Re\left\{r_{n} \cdot s_{n}^{*}(\mathbf{p})\right\}\right\} .
$$

When using a traditional array, $s_{n}(\mathbf{p})$ is given by (6), whereas with a lens antenna it is expressed by the first term of (20).

\section{RESUlts}

We now evaluate the position estimation performance according to the previously performed analysis. More specifically, in our scenario we account only of the LOS component. Then, we consider a transmitter sending pulses centered at $f_{0}=60 \mathrm{GHz}$, with a bandwidth $W=4 \mathrm{GHz}$ and an effective radiated isotropic power (EIRP) of $20 \mathrm{dBm}$. At the receiver, we account for a noise figure $F=4 \mathrm{~dB}$. Parameter $A_{\mathrm{pl}}$ in (5) is obtained from the link budget.

Then, we alternatively consider a lens-embedded array and a traditional linear uniform array with different number $N_{\mathrm{A}}$ of antennas (i.e., 51, 101 and 201) and, thus, physical length $D_{z}$ (i.e., $12.5 \mathrm{~cm}, 25 \mathrm{~cm}$ and $5 \mathrm{~cm}$ ). Note that arrays with $N_{\mathrm{A}}=51$ and $N_{\mathrm{A}}=101$ antennas have a size suitable for their integration in portable devices, whereas uniform linear arrays with $N_{\mathrm{A}}=201$ still preserve a length shorter than $1 \mathrm{~m}$. Note that in case of the lens, a physical aperture of $D_{y}=D_{z} / 4$ along the $y$-axis has been accounted for. Notably, in agreement with the analysis and comparison of the architectures conducted in [7], this implies a higher normalized aperture $\mathcal{A}$ when the lens is considered, thanks to its capacity to collect and collimate the wavefront of the impinging wave.

Results are expressed in terms of the root mean square error (RMSE) of the position estimate, which is evaluated as

$$
\operatorname{RMSE}(\hat{\mathbf{p}})=\sqrt{\frac{1}{N_{\mathrm{c}}} \sum_{i=1}^{N_{\mathrm{c}}}\left\|\hat{\mathbf{p}}_{i}-\mathbf{p}\right\|^{2}}
$$

where $N_{\mathrm{c}}$ is the number of Monte Carlo iterations considered in simulations and $\hat{\mathbf{p}}_{i}$ is the position estimate at the $i$ th iteration. For each cycle, a different noise realization is generated according to $\sigma^{2}$, and a different realization of phase $\chi$ is drawn, which is kept the same for all the antennas. In this way, random phase models a complete clock mismatch between the transmitter and the receiver.

Fig. 4 reports the obtained results with and without the lens (i.e., traditional arrays), and for different $N_{\mathrm{A}}$. We initially fixed the AOA to $0^{\circ}$ by varying only the TX-RX distance from $5 \mathrm{~m}$ to $30 \mathrm{~m}$. As expected, the larger is $N_{\mathrm{A}}$, the better is the position estimate thanks to the increased physical length. In addition, the use of a lens allows to improve the achievable performance, thanks to the increased normalized aperture once $N_{\mathrm{A}}$ is fixed [7]. This effect is more pronounced for larger distances, where the path loss increases. In fact, as an example, by using a lens with $N_{\mathrm{A}}=101$, the positioning error is kept at about $2 \mathrm{~m}$ for $d=30 \mathrm{~m}$. Instead, for its counterpart without the lens, the positioning error is higher and reaches $5 \mathrm{~m}$ at $d=30 \mathrm{~m}$. Finally, it is important to remark that localization errors below $1 \mathrm{~m}$ for $d=30 \mathrm{~m}$ can be attained with a physical length of 


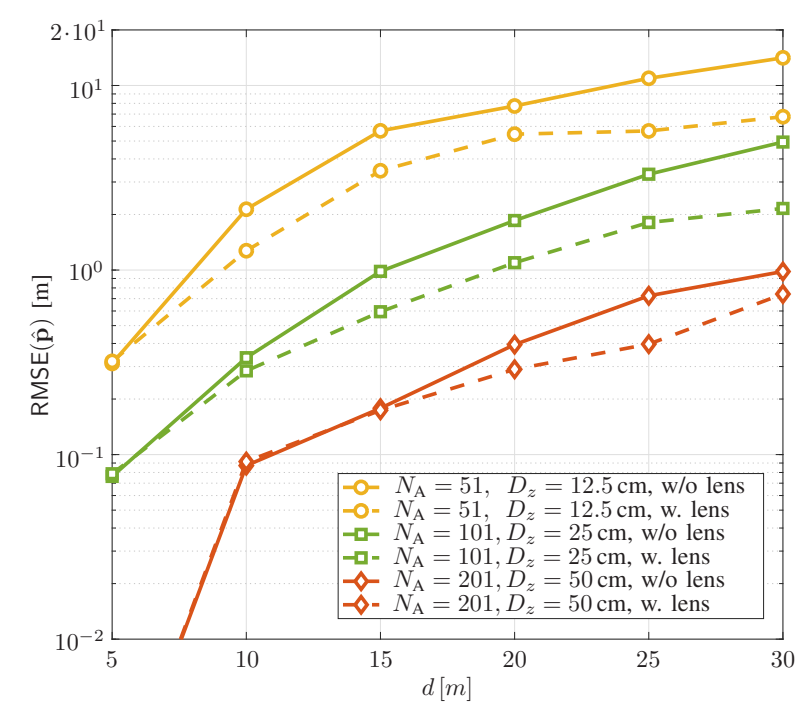

Fig. 4. RMSE as a function of the TX-RX distance $d$, fixed $\mathrm{AOA}=0^{\circ}$ and different array size (i.e., different number of antennas $N_{\mathrm{A}}$ ).

at most $50 \mathrm{~cm}$, which is still practical in real scenarios, for example when employed in access points.

Fig. 5 reports instead results achieved for $\mathrm{AOA}=0^{\circ}$, $\mathrm{AOA}=30^{\circ}$ and $\mathrm{AOA}=60^{\circ}$ when $N_{\mathrm{A}}$ is fixed to 101 . More specifically, it is evidenced that for an AOA of $60^{\circ}$ performance are substantially degraded with respect to $30^{\circ}$, where the RMSE is about $2.5 \mathrm{~m}$ for $d=30 \mathrm{~m}$ and when the lens is used. Note that, for the considered link budget, position errors of at most $2 \mathrm{~m}$ can be guaranteed until $d=20 \mathrm{~m}$ for an $\mathrm{AOA}$ of $0^{\circ}$ and $30^{\circ}$ and when using a lens array.

The results herein reported denote that compact array architectures with at most 201 antennas, i.e., with a diameter of less than $50 \mathrm{~cm}$, can be exploited to guarantee accurate positioning performance exploiting only the wavefront curvature and using only a single antenna array, without letting the transmitter and receiver be synchronous.

\section{CONCLUSIONS}

In this paper, the possibility to directly infer the transmitter position from the impinging wavefront curvature at mm-wave has been investigated. The analysis has been conducted for arrays at $60 \mathrm{GHz}$, equipped with and without a lens, showing the clear advantage of using lens to reduce the number of antenna elements or improve the performance. The outcomes of this paper show that this solution allows source localization using a single antenna array without transmitter and receiver interaction and synchronization.

\section{ACKNOWLEDGMENTS}

This work has been in part funded by the European Commission through the framework of the H2020 project XCycle under Grant 635975.

\section{REFERENCES}

[1] F. Boccardi et al., "Five disruptive technology directions for 5G," IEEE Commun. Mag., vol. 52, no. 2, pp. 74-80, Feb. 2014.

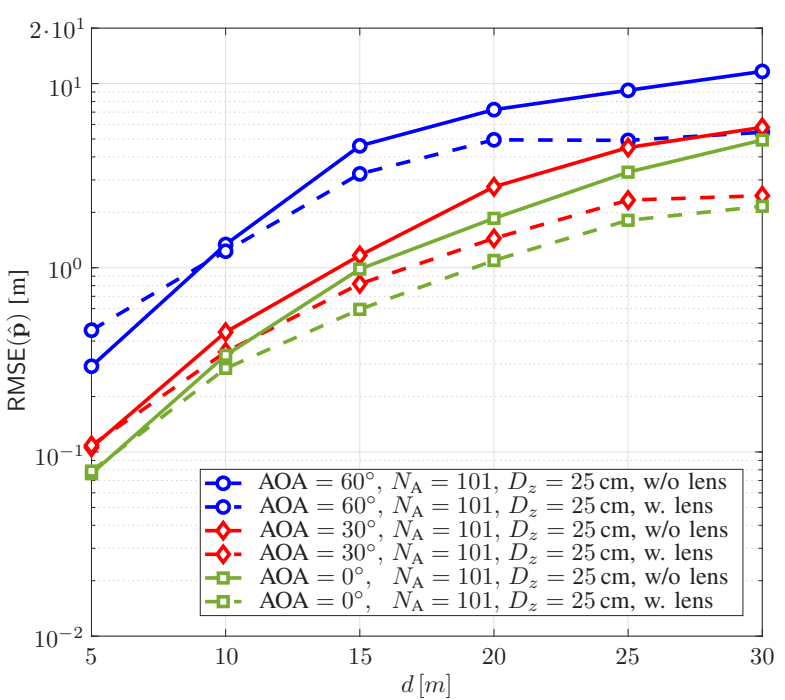

Fig. 5. RMSE as a function of the TX-RX distance $d$ for fixed $N_{\mathrm{A}}=101$ and for different AOA.

[2] W. Roh et al., "Millimeter-wave beamforming as an enabling technology for $5 \mathrm{G}$ cellular communications: theoretical feasibility and prototype results," IEEE Commun. Mag., vol. 52, no. 2, pp. 106-113, 2014.

[3] F. Guidi, A. Guerra, and D. Dardari, "Personal mobile radars with millimeter-wave massive arrays for indoor mapping," IEEE Trans. Mobile Comput., vol. 15, no. 6, pp. 1471-1484, Jun. 2016.

[4] F. Guidi et al., "Joint energy detection and massive array design for localization and mapping," IEEE Trans. Wireless Commun., vol. 16, no. 3, pp. 1359-1371, Mar. 2017.

[5] A. F. Molisch et al., "Hybrid beamforming for massive MIMO: A survey," IEEE Commun. Mag., vol. 55, no. 9, 2017.

[6] A. Guerra, F. Guidi, and D. Dardar, "Single anchor localization and orientation performance limits using massive arrays: MIMO vs. beamforming," IEEE Trans. Wireless Commun., vol. XX, no. XX, p. XX, 2018.

[7] Y. Zeng and R. Zhang, "Millimeter wave MIMO with lens antenna array: A new path division multiplexing paradigm," IEEE Trans. Commun., vol. 64, no. 4, pp. 1557-1571, Apr. 2016.

[8] Y. Zeng, R. Zhang, and Z. N. Chen, "Electromagnetic lens-focusing antenna enabled massive MIMO: Performance improvement and cost reduction," IEEE J. Sel. Areas Commun., vol. 32, no. 6, pp. 1194-1206, 2014.

[9] F. Guidi, "AOA estimation with EM lens-embedded massive arrays," in Proc. IEEE Int. Veh. Tech. Conf. (VTC), Porto, Portugal, Jun. 2018.

[10] J. Liang and D. Liu, "Passive localization of mixed near-field and farfield sources using two-stage MUSIC algorithm," IEEE Trans. Signal Processing, vol. 58, no. 1, pp. 108-120, Jan. 2010.

[11] N. Hadaschik, B. Sackenreuter, and M. Fa?binder, "Direct multi-array and multi-tone positioning," in Proc. IEEE Int. Conf. on Commun. Workshops (ICC Workshops), May 2017, pp. 1067-1072.

[12] M. N. E. Korso et al., "Sequential estimation of the range and the bearing using the Zero-Forcing Music approach," in Proc. 17th European Signal Processing Conf. (EUSIPCO), Aug. 2009, pp. 1404-1408.

[13] — , "Deterministic performance bounds on the mean square error for near field source localization," IEEE Trans. Signal Processing, vol. 61, no. 4, pp. 871-877, Feb. 2013.

[14] J.-P. Le Cadre, "Performance analysis of wavefront curvature methods for range estimation of a moving source," IEEE Trans. Aerosp. Electron. Syst., vol. 31, no. 3, pp. 1082-1103, 1995.

[15] B. G. Ferguson and R. J. Wyber, "Wavefront curvature passive ranging in a temporally varying sound propagation medium," in Proc. MTS/IEEE Oceans. An Ocean Odyssey, vol. 4, 2001, pp. 2359-2365 vol.4.

[16] P. Stoica and R. L. Moses, Spectral analysis of signals. PRENTICE HALL, 2005, vol. 1. 